\title{
Low Intensity Electrical Observer Effect
}

\author{
Sultan Muhammad \\ Qurtuba University Phase 3 Hayatabad Peshawar, Pakistan
}

\section{*Correspondence author}

Sultan Muhammad

Qurtuba University Phase 3 Hayatabad Peshawar, Pakistan

Submitted : 15 Feb 2021 ; Published : 3 Mar 2021

\begin{abstract}
The study of the dual nature of the electron is proposed, with alterations in initial double-slit experiment. We are aware of mysterious nature of the quantum particles, particularly electrons. The particle behaves as wave, but upon observation, the wave-function of the electron is collapsed. No longer is an interference pattern observed, which in a sense, limits us and binds us to what we can measure and what we cannot, with current detection methods. In the double slit experiment, a source of high-intensity light is used to measure the position of the electrons, which leads to the collapse of the wave-function. An investigation is proposed to observe the effects of the low-intensity electric field on the duality of electron.
\end{abstract}

Keywords: Double-slit experiment, Quantum duality, Measurements, QED, Electron

\section{Introduction}

The infamous double slit experiment [1] is the heart of quantum mechanics (Feynman). The experiment produces variable results, depending on the measurements to be taken. If we let the beam of electrons pass through the two slits, interference pattern is detected $[2,4]$ which is a fundamental property of the waves. But a riveting phenomenon occurs when we try to measure the position of the electron to authenticate from which slit the electron went through; interference pattern collapses [5 7]. The very act of measuring can change the state of the free electron. In the double slit experiment, a high-intensity light source is used as a detector, which interferes with the state of electron [10]. The duality of electron is also linked to De Broglie hypothesis [8]. There is another phenomena involved, such as Compton Effect [9] which transpires while measuring the position of the electron.

A recent mechanism was proposed to explain the interaction of the detector's photons with the free electron, [10] and to elucidate the role of observer. From all the arguments, it becomes distinct that we cannot use light to measure the position of free electrons [11]. Thus, a new approach is postulated, with slight modifications to the apparatus of the double slit experiment. With this modification, we might be able to measure the position of the free electron, without interfering with the dual nature of the electron. We intend to use electric field, instead of photons, to measure the position of the free electrons. This will confirm whether detector (electrical field) collapses the wave-function of electron, like the photons do, or not.

\section{Hypothesis}

We know that measurement causes the wave function of the electron to collapse, as the photons are used as detector. We also understand that Photons are packets of energy $[12,13]$. This quantized packet of energy can and will interact with other quantum particles, in this case, the free electrons. Thus, the detection of the path of the electron factors the wave function to collapse. The goal is to possibly create such detectors that can measure the position of the free electron, without collapsing the wave function $[14,15]$ of the electrons. So, we propose a prototype to investigate the effects of low-intensity electric fields on the wave-function of the electrons.

\section{Theoretical and practical framework}

We can produce free electrons by using Cathode ray tube (CRT's) $[16,17]$ and electron gun $[18,19]$ to accelerate the electrons, and confine them into a beam. Whenever a charged particle approaches an electrical field, it experiences a drag force that propels it towards and away from the positive to negative plates, respectively. The force is calculated by formula:

$$
\vec{F}=q \vec{E}
$$

Where " $F$ " is the force experienced by the particle,

"q" is the charge of the particle and

"E" is electrical field intensity.

Figure (1) shows CRT and accelerating mechanism of an electron gun 
Figure (1) shows CRT and accelerating mechanism of an electron gun

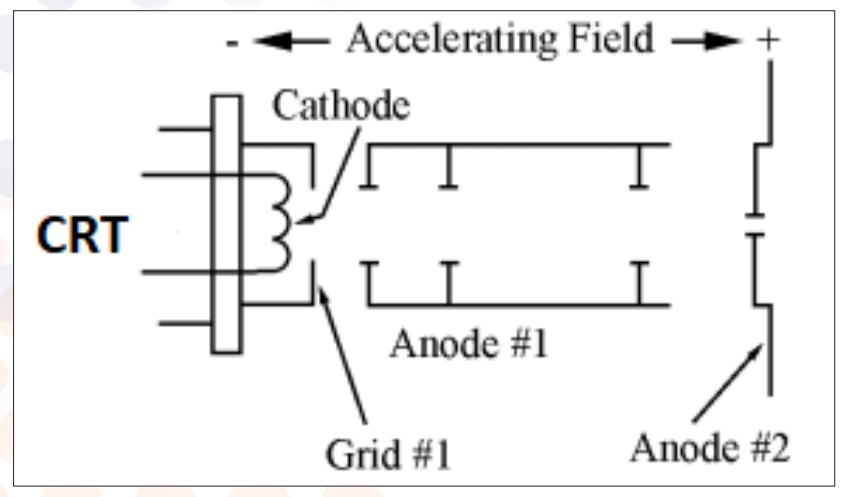

Figure 1

When potential difference is applied to CRT, the electrons are kicked out of atoms. These free electrons are then subjected to a electrical field. This electrical field is just for acceleration. The key to the experiment is to introduce a non-parallel electrical field. The applied field must be perpendicular to the path of the beam, so that the path of the beam is curved.

The velocity of the electron leaving the electron gun can be calculated

As the kinetic energy is gained by the action of potential difference

$$
\begin{aligned}
& K E=\frac{1}{2} m_{e} v^{2}---(2) \\
& E=e V---(2.1)
\end{aligned}
$$

By comparing equation (2) with equation (2.1),

$$
\begin{aligned}
& \frac{1}{2} m_{e} v^{2}=e V--(2.3) \\
& v^{2}=\frac{2 e V}{m_{e}}--(2.4) \\
& v=\sqrt{\frac{2 e V}{m_{e}}}-- \text { (3) }
\end{aligned}
$$

The equation (3) represents the velocity of the electrons leaving the electron gun.

Where "e" is the charge of electron [20 22],

"V" is the applied voltage and

"me" is the mass of the electron [23 25].

As the electrons are accelerated, we now need to introduce an electrical field to bend the beam of the electron, so that we can conform the interaction between the particle and the introduced field. A uniform electric field is introduced, perpendicular to the motion of the beam of electrons. Figure (2) illustrates the phenomena:

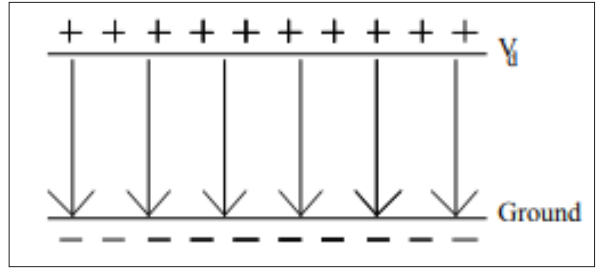

Figure 2

The field strength is given as:

$$
E_{\circ}=\frac{k q}{d^{2}}--(4)
$$

As the electric field is constant, we can use equation:

$$
E_{\circ}=\frac{V}{d}--(4.1)
$$

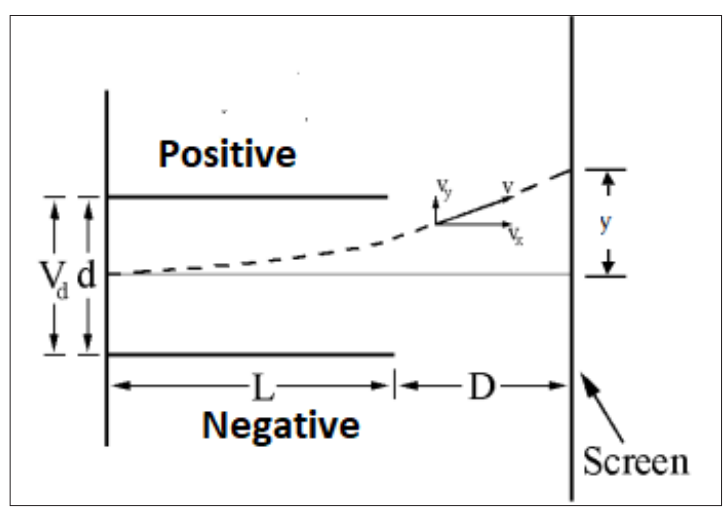

Figure 3

By using kinematics and new $2^{\text {nd }}$ equation of motion, we can calculate the deviation of the path of electron, due to the induced electric field:

$$
Y=\frac{e E L}{m v^{2}}\left(D+\frac{L}{2}\right)---(5)
$$

As the induced electric field is perpendicular to the path of electron:

$$
\begin{aligned}
& E_{\circ}=\frac{V}{d}--(4.1) \\
& Y=\frac{e V L}{m v^{2} d}\left(D+\frac{L}{2}\right)---(6)
\end{aligned}
$$

Now, we modify the apparatus and swap the screen with the slits and at a distance, then we utilize a screen.

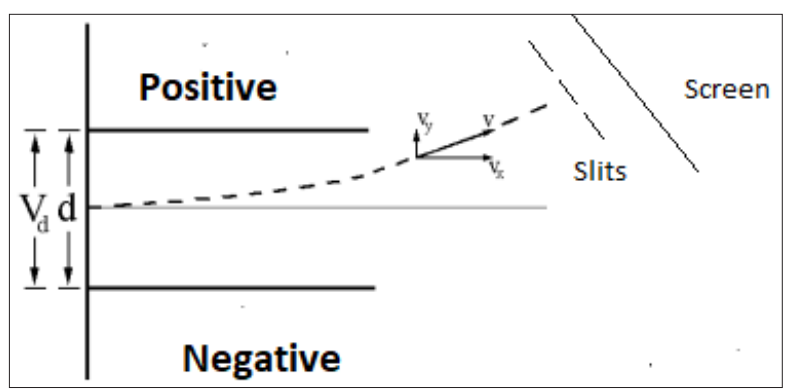

\section{Figure 4}

As the study involves low-intensity electric fields, we are restricted to low voltages only

$$
\lim V_{1}^{100}
$$




\section{Conclusion}

From the above apparatus design, we can investigate the effects of Low-intensity electric fields on the nature of the electron. If the interference pattern disappears, we will conclude that electric field may not be utilized to measure the position of the electron. But, if the interference pattern is consistent, then the only conclusion is that the low-intensity electric field does not interfere with the nature of the electron. Hence we might be able to theorize a special class of detectors to measure the position without interfering the nature of particular quantum entity.

\section{Discussions}

The proposed design of the double slit experiment is a unique approach. If we succeed in the preservation of the wave function of the free electrons, it could open a whole new chapter for us to discover and the way we measure physical entities, such as quantum particles.

\section{Acknowledgements}

I offer concise but sincere acknowledgements to Prof Dr. Fazal-ur-Rehman Sethi (supervisor), Syeda Omama Jamshaid, Maham Tariq (Major contributor) and all those who offered their wisdom and time in my research.

\section{References}

1. Jönsson C (1961) Elektroneninterferenzen a mehreren künstlich hergestellten Feinspalten. Zeitschrift für Physics 161: 454-474.

2. Tonomura A, Endo J, Matsuda T, Kawasaki T, Ezawa H (1989) Demonstration of single-electron buildup of an interference pattern. American Journal of Physics, 57: 117120.

3. Bach R, Pope D, Liou SH, Batelaan H (2013) Controlled double-slit electron diffraction. New Journal of Physics 15: 033018 .

4. Frabboni S, Gabrielli A, Gazzadi GC, Giorgi F, Matteucci G, etal (2012) The Young-Feynman two-slits experiment with single electrons: Build-up of the interference pattern and arrival-time distribution using a fast-readout pixel detector. Ultramicroscopy 116: 73-76.

5. Zou XY, Wang LJ, Mandel L (1991) Induced coherence and in distinguishability in optical interference. Physical review letters 67: 318 .

6. Buks E, Schuster R, Heiblum M, Mahalu D, Umansky V (1998) Dephasing in electron interference by a 'which-path'detector. Nature 391: 871-874.

7. Neder I, Marquardt F, Heiblum M, Mahalu D, Umansky V (2007) Controlled dephasing of electrons by non-gaussian shot noise. Nature Physics 3: 534-537.

8. De Broglie L (1923) Waves and quanta. Nature, 112: 540540.

9. Williams B (1977) Compton scattering: the investigation of electron momentum distributions.

10. Muhammad S, Omama M (2020) A New Approach to Du- ality of Electron. Energy, 14: 16.

11. Zel'Dovich YB (1975) Interaction of free electrons with electromagnetic radiation Soviet Physics Uspekhi 18: 79.

12. Pratt RH, Ron A, Tseng HK (1973) Atomic photoelectric effect above $10 \mathrm{keV}$ Reviews of Modern physics 45: 273.

13. Colladay D, McDonald P, Potting R (2014) Gupta-Bleuler photon quantization in the standard model extension. Physical Review D, 89: 085014.

14. Bassi A, Lochan K, Satin S, Singh TP, Ulbricht H (2013) Models of wave-function collapse, underlying theories, and experimental tests. Reviews of Modern Physics 85: 471.

15. Namiki M, Pascazio S (1991) Wave-function collapse by measurement and its simulation. Physical Review A 44: 39.

16. Tomihari Y (1998) U.S. Patent No. 5,719,477. Washington, DC: U.S. Patent and Trademark Office.

17. Jang YT, Lee YH, Ju BK, Ahn JH, Go CK, etal. (2002) Application of carbon nanotubes to the cathode ray tube-electron gun. Vacuum, 68: 79-85.

18. Crewe AV, Eggenberger DN, Wall J, Welter LM (1968) Electron gun using a field emission source. Review of Scientific Instruments 39: 576-583.

19. Westenskow GA, Madey JMJ (1984) Microwave electron gun. Laser and Particle Beams, 2: 223-225.

20. Westenskow GA, Madey JMJ (1984) Microwave electron gun. Laser and Particle Beams 2: 223-225.

21. Allen MD, Raabe OG, (1985) Slip correction measurements of spherical solid aerosol particles in an improved Millikan apparatus. Aerosol Science and Technology 4: 269-286.

22. Mohr P J, Taylor B N (2005) CODATA recommended values of the fundamental physical constants: 2002. Reviews of modern physics, 77- 81.

23. Farnham DL, Van Dyck Jr, RS Schwinberg PB (1995) Determination of the electron's atomic mass and the proton/ electron mass ratio via Penning trap mass spectroscopy. Physical review letters, 75:3598.

24. Gainutdinov RK, Khamadeev MA, Salakhov MK (2012) Electron rest mass and energy levels of atoms in the photonic crystal medium. Physical Review A, 85: 053836.

25. Sukhoruchkin S (2017) Confirmation of the discreteness in particle masses and the SM parameters, Nuclear and particle physics proceedings 282: 189-193.

Copyright: (C2021 Sultan Muhammad. This is an open-access article distributed under the terms of the Creative Commons Attribution License, which permits unrestricted use, distribution, and reproduction in anymedium, provided the original author and source are credited. 